\title{
On the Nonlinear Difference Equation
}

\author{
Elmetwally M. Elabbasy, Abdulmuhaemn A. El-Biaty \\ Department of Mathematics, Faculty of Science, Mansoura University, Mansoura, Egypt \\ Email: emelabbasy@mans.edu.eg, aaalbayaty77@gmail.com
}

Received 17 November 2015; accepted 22 January 2016; published 26 January 2016

Copyright (C) 2016 by authors and Scientific Research Publishing Inc.

This work is licensed under the Creative Commons Attribution International License (CC BY).

http://creativecommons.org/licenses/by/4.0/

(c) (i) Open Access

\section{Abstract}

In this paper, we investigate some qualitative behavior of the solutions of the difference equation $x_{n+1}=a+\frac{b x_{n-k}}{\sum_{i=0}^{k} c_{i} x_{n-i}}, n=0,1,2, \cdots$, where the coefficients $a, b$ and $c_{i}$ are positive real numbers, $i, k \in\{0,1,2, \cdots\}$ and where the initial conditions $x_{-k}, x_{-k+1}, \cdots, x_{0}$ are arbitrary positive real numbers.

\section{Keywords}

Difference Equation, Stability, Periodicity, Boundedness, Global Stability

\section{Introduction}

Our aim in this paper is to study with some properties of the solutions of the difference equation

$$
x_{n+1}=a+\frac{b x_{n-k}}{\sum_{i=0}^{k} c_{i} x_{n-i}}, \quad n=0,1,2, \cdots,
$$

where the coefficients $a, b$ and $c_{i}$ are positive real numbers, $i, k \in\{0,1,2, \cdots\}$ and where the initial conditions $x_{-k}, x_{-k+1}, \cdots, x_{0}$ are arbitrary positive real numbers. There is a class of nonlinear difference equations, known as the rational difference equations, each of which consists of the ratio of two polynomials in the sequence terms in the same form. There has been a lot of work concerning the global asymptotics of solutions of rational difference equations [1]-[8].

Many researchers have investigated the behavior of the solution of difference equation. For example:

Amleh et al. [9] has studied the global stability, boundedness and the periodic character of solutions of the equation 


$$
x_{n+1}=\alpha+\frac{x_{n-1}}{x_{n}} .
$$

Our aim in this paper is to extend and generalize the work in [9], [10] and [11]. That is, we will investigate the global behavior of (1.1) including the asymptotical stability of equilibrium points, the existence of bounded solution, the existence of period two solution of the recursive sequence of Equation (1).

Now we recall some well-known results, which will be useful in the investigation of (1.1) and which are given in [12].

Let $I$ be an interval of real numbers and let

$$
F: I^{k+1} \rightarrow I
$$

where $F$ is a continuous function. Consider the difference equation

$$
y_{n+1}=F\left(y_{n}, y_{n-1}, \cdots, y_{n-k}\right), \quad n=0,1,2, \cdots,
$$

with the initial condition $y_{-k}, y_{-k+1}, \cdots, y_{0} \in I$.

Definition 1. (Equilibrium Point)

A point $\bar{y} \in I$ is called an equilibrium point of Equation (1.2) if

$$
\bar{y}=f(\bar{y}, \bar{y}, \cdots, \bar{y}) \text {. }
$$

That is, $y_{n}=\bar{y}$ for $n \geq 0$, is a solution of Equation (1.2), or equivalently, $\bar{y}$ is a fixed point of $f$.

Definition 2. (Stability)

Let $\bar{y} \in(0, \infty)$ be in equilibrium point of Equation (1.2) then

1) An equilibrium point $\bar{y}$ of Equation (1.2) is called locally stable if for every $\varepsilon>0$ there exists $\delta>0$ such that, if $y_{-k}, y_{-k+1}, \cdots, y_{0} \in(0, \infty)$ with $\left|y_{-k}-\bar{y}\right|+\left|y_{-k+1}-\bar{y}\right|+\cdots+\left|y_{0}-\bar{y}\right|<\delta$, then $\left|y_{n}-\bar{y}\right|<\varepsilon$ for all $n \geq-k$.

2) An equilibrium point $\bar{y}$ of Equation (1.2) is called locally asymptotically stable if $\bar{y}$ is locally stable and there exists $\gamma>0$ such that, if $y_{-k}, y_{-k+1}, \cdots, y_{0} \in(0, \infty)$ with

$$
\left|y_{-k}-\bar{y}\right|+\left|y_{-k+1}-\bar{y}\right|+\cdots+\left|y_{0}-\bar{y}\right|<\gamma \text {, then } \lim _{n-\infty} y_{n}=\bar{y}
$$

3) An equilibrium point $\bar{y}$ of Equation (1.2) is called a global attractor if for all $y_{-k}, y_{-k+1}, \cdots, y_{0} \in(0, \infty)$ we have

$$
\lim _{n \rightarrow \infty} y_{n}=\bar{y}
$$

4) An equilibrium point $\bar{y}$ of Equation (1.2) is called globally asymptotically stable if $\bar{y}$ is locally stable and a global attractor.

5) An equilibrium point $\bar{y}$ of Equation (1.2) is called unstable if $\bar{y}$ is not locally stable.

Definition 3. (Permanence)

Equation (1.2) is called permanent if there exists numbers $m$ and $M$ with $0<m<M<\infty$ such that for any initial conditions $y_{-k}, y_{-k+1}, \cdots, y_{0} \in(0, \infty)$ there exists a positive integer $N$ which depends on the initial conditions such that

$$
m \leq y_{n} \leq M \text { for all } n \geq-k
$$

Definition 4. (Periodicity)

A sequence $\left\{x_{n}\right\}_{n=-k}^{\infty}$ is said to be periodic with period $p$ if $x_{n+p}=x_{n}$ for all $n \geq-k$. A sequence $\left\{x_{n}\right\}_{n=-k}^{\infty}$ is said to be periodic with prime period $p$ if $p$ is the smallest positive integer having this property.

The linearized equation of Equation (1.2) about the equilibrium point $\bar{y}$ is defined by the equation

$$
z_{n+1}=\sum_{i=0}^{k} p_{i} z_{n-i},
$$

where 


$$
p_{i}=\frac{\partial F(\bar{y}, \bar{y}, \cdots, \bar{y})}{\partial y_{n-i}}, \quad i=0,1, \cdots, k .
$$

The characteristic equation associated with Equation (1.3) is

$$
\lambda^{k+1}-p_{0} \lambda^{k}-p_{1} \lambda^{k-1}-\cdots-p_{k-1} \lambda-p_{k}=0 .
$$

Theorem 1.1. [13] Let $[p, q]$ be an interval of real numbers and assume that

$$
g:[p, q]^{k+1} \rightarrow[p, q]
$$

is a continuous function satisfying the following properties:

(a) $g\left(x_{1}, x_{2}, \cdots, x_{k+1}\right)$ is non-increasing in the first $(k)$ terms for each $x_{k+1}$ in $[p, q]$ and non-decreasing in the last term for each $x_{i}$ in $[p, q]$ for all $i=1,2, \cdots, k$.

(b) If $(m, M) \in[p, q] \times[p, q]$ is a solution of the system

$$
M=g(m, m, m, \cdots, m, M) \text { and } m=g(M, M, M, \cdots, M, m),
$$

implies

$$
m=M \text {. }
$$

Theorem 1.2. [12] Assume that $F$ is a $C^{1}$-function and let $\bar{y}$ be an equilibrium point of Equation (1.2). Then the following statements are true:

1) If all roots of Equation (1.4) lie in the open unit disk $|\lambda|<1$, then he equilibrium point $\bar{y}$ is locally asymptotically stable.

2) If at least one root of Equation (1.4) has absolute value greater than one, then the equilibrium point $\bar{y}$ is unstable.

3) If all roots of Equation (1.4) have absolute value greater than one, then the equilibrium point $\bar{y}$ is a source.

Theorem 1.3. [14] Assume that $p_{i} \in R, i=1,2, \cdots, k$. Then

$$
\sum_{i=1}^{k}\left|p_{i}\right|<1
$$

is a sufficient condition for the asymptotically stable of Equation (1.5)

$$
y_{n+k}+p_{1} y_{n+k-1}+\cdots+p_{k} y_{n}=0, n=0,1, \cdots .
$$

\section{Local Stability of Equation (1.1)}

In this section we investigate the local stability character of the solutions of Equation (1.1). Equation (1.1) has a unique nonzero equilibrium point

$$
\begin{aligned}
& \bar{x}=a+\frac{b \bar{x}}{\sum_{i=0}^{k} \bar{c} \bar{x}}, \\
& \bar{x}=a+\frac{b}{\sum_{i=0}^{k} c_{i}} .
\end{aligned}
$$

Let

$$
G=\sum_{i=0}^{k} c_{i}
$$

Then, we get

$$
\bar{x}=a+\frac{b}{G} .
$$

Let $f:(0, \infty)^{k+1} \rightarrow(0, \infty)$ be a function defined by 


$$
f\left(u_{0}, u_{1}, \cdots, u_{k}\right)=a+\frac{b u_{k}}{\sum_{i=0}^{k} c_{i} u_{i}}
$$

Therefore it follows that

$$
\frac{\partial f\left(u_{0}, u_{1}, \cdots, u_{k}\right)}{\partial u_{j}}=\frac{-b u_{k} c_{j}}{\left[\sum_{i=0}^{k} c_{i} u_{i}\right]^{2}}, \quad j=0,1, \cdots, k-1,
$$

and

$$
\frac{\partial f\left(u_{0}, u_{1}, \cdots, u_{k}\right)}{\partial u_{k}}=b \frac{\sum_{i=0}^{k-1} c_{i} u_{i}}{\left[\sum_{i=0}^{k} c_{i} u_{i}\right]^{2}} .
$$

Then we see that

$$
\frac{\partial f\left(\bar{x}, \cdots, \bar{x}_{k}\right)}{\partial u j}=-b \frac{c_{j}}{(a G+b) G}=-P_{j}, j=0,1, \cdots, k-1,
$$

and

$$
\frac{\partial f\left(\bar{x}, \cdots, \bar{x}_{k}\right)}{\partial u_{k}}=b \frac{G-c_{k}}{(a G+b) G}=-P_{k} .
$$

Then the linearized equation of (1.1) about $\bar{x}$ is

$$
z_{n+1}=\sum_{i=0}^{k} p_{i} z_{n-i} \text {. }
$$

Theorem 2.1. Assume that

$$
(b-a G) G<2 b c_{k} \text {. }
$$

Then the equilibrium point of Equation (1.1) is locally stable.

Proof. It is follows by Theorem (1.3) that, Equation (2.2) is locally stable if

$$
\left|p_{k}\right|+\cdots+\left|p_{1}\right|+\left|p_{0}\right|<1 \text {. }
$$

That is

$$
\left|\frac{b c_{0}}{(a G+b) G}\right|+\left|\frac{b c_{1}}{(a G+b) G}\right|+\cdots+\left|\frac{b c_{k-1}}{(a G+b) G}\right|+\left|\frac{b\left(G-c_{k}\right)}{(a G+b) G}\right|<1 .
$$

This implies that

$$
\frac{b\left[\sum_{i=0}^{k-1} c_{i}\right]}{(a G+b) G}+\left|\frac{b\left(G-c_{k}\right)}{(a G+b) G}\right|<1,
$$

then

$$
\frac{2 b\left(G-c_{k}\right)}{(a G+b) G}<1
$$

Thus

$$
(b-a G) G<2 b c_{k}
$$

Hence, the proof is completed. 


\section{Periodic Solutions}

In this section we investigate the periodic character of the positive solutions of Equation (1.1).

Theorem 3.1. Equation (1.1) has positive prime period-two solution only if

$$
k-o d d \text { and }(\alpha-\beta)(a \beta-a \alpha+b)>4 a \alpha \beta .
$$

Proof. Assume that there exists a prime period-two solution

$$
\cdots, p, q, p, q, \cdots
$$

of (1.1). Let $x_{n}=q, x_{n+1}=p$. Since $k$-odd, we have $x_{n-k}=p$. Thus, from Equation (1.1), we get

$$
p=a+\frac{b p}{c_{0} q+c_{1} p+c_{2} q+\cdots+c_{k} p}
$$

and

$$
q=a+\frac{b q}{c_{0} p+c_{1} q+c_{2} p+\cdots+c_{k} q}
$$

Let

$$
c_{0}+c_{2}+\cdots+c_{k-1}=\alpha
$$

and

$$
c_{1}+c_{3}+\cdots+c_{k}=\beta .
$$

Then

$$
p=a+\frac{b p}{\alpha q+\beta p},
$$

and

$$
q=a+\frac{b q}{\alpha p+\beta q} .
$$

Then

$$
\alpha p q+\beta p^{2}=a \alpha q+a \beta p+b p,
$$

and

$$
\alpha p q+\beta q^{2}=a \alpha p+a \beta q+b q .
$$

Subtracting (3.2) from (3.3) gives

$$
\beta\left(p^{2}-q^{2}\right)=(a \beta-a \alpha+b)(p-q) .
$$

Since $p \neq q$, we have

$$
p+q=\frac{a \beta-a \alpha+b}{\beta} .
$$

Also, since $p$ and $q$ are positive, $(a \beta-a \alpha+b)$ should be positive. Again, adding (3.2) and (3.3) yields

$$
2 \alpha p q+\beta\left(p^{2}+q^{2}\right)=(a \alpha+a \beta+b)(p+q) .
$$

It follows by (3.4), (3.5) and the relation

$$
p^{2}+q^{2}=(p+q)^{2}-2 p q, \quad \forall p, q \in \mathrm{R},
$$

that

$$
p q=\frac{a \alpha(a \beta-a \alpha+b)}{\beta(\alpha-\beta)} .
$$


Assume that $p$ and $q$ are two distinct real roots of the quadratic equation

$$
\beta t^{2}-(a \beta-a \alpha+b) t+\frac{a \alpha(a \beta-a \alpha+b)}{(\alpha-\beta)}=0,
$$

and so

$$
(a \beta-a \alpha+b)^{2}-4 \beta \frac{a \alpha(a \beta-a \alpha+b)}{(\alpha-\beta)}>0,
$$

which is equivalent to

$$
(\alpha-\beta)(a \beta-a \alpha+b)>4 a \alpha \beta
$$

Thus, the proof is completed.

\section{Bounded Solution}

Our aim in this section we investigate the boundedness of the positive solutions of Equation (1.1).

Theorem 4.1. The solutions $\left\{x_{n}\right\}_{n=-k}^{\infty}$ of Equation (1.1) are bounded.

Proof. Let $\left\{x_{n}\right\}_{n=-k}^{\infty}$ be a solution of Equation (1.1). We see from Equation (1.1) that

$$
x_{n+1}=a+\frac{b x_{n-k}}{\sum_{i=0}^{k} c_{i} x_{n-i}}=a+\frac{b x_{n-k}}{c_{0} x_{n}+c_{1} x_{n-1}+\cdots+c_{k} x_{n-k}} .
$$

Then

$$
x_{n} \leq a+\frac{b}{c_{k}}=M \text { for all } n \geq 1 .
$$

On the other hand, we see that the change of variables

$$
x_{n}=\frac{1}{y_{n}},
$$

transforms Equation (1.1) to the following form:

$$
\frac{1}{y_{n+1}}=a+\frac{\frac{b}{y_{n-k}}}{\frac{c_{0}}{y_{n}}+\frac{c_{1}}{y_{n-1}}+\cdots+\frac{c_{k}}{y_{n-k}}} .
$$

Hence, we obtain

$$
\frac{1}{y_{n+1}}=a+\frac{\frac{b}{y_{n-k}} \prod_{i=0}^{k} y_{n-i}}{c_{0} \prod_{i=1}^{k} y_{n-i}+c_{1} \prod_{i=0, i \neq 1}^{k} y_{n-i}+\cdots+c_{k} \prod_{i=0}^{k-1} y_{n-i}} .
$$

Thus

$$
\begin{gathered}
y_{n+1}=\frac{c_{0} \prod_{i=1}^{k} y_{n-i}+c_{1} \prod_{i=0, i \neq 1}^{k} y_{n-i}+\cdots+c_{k} \prod_{i=0}^{k-1} y_{n-i}}{a c_{0} \prod_{i=1}^{k} y_{n-i}+a c_{1} \prod_{i=0, i \neq 1}^{k} y_{n-i}+\cdots+\left(a c_{k}+b\right) \prod_{i=0}^{k-1} y_{n-i}}=\frac{c_{0} \prod_{i=1}^{k} y_{n-i}}{a c_{0} \prod_{i=1}^{k} y_{n-i}+a c_{1} \prod_{i=0, i \neq 1}^{k} y_{n-i}+\cdots+\left(a c_{k}+b\right) \prod_{i=0}^{k-1} y_{n-i}} \\
+\frac{c_{1} \prod_{i=0, i \neq 1}^{k} y_{n-i}}{a c_{0} \prod_{i=1}^{k} y_{n-i}+a c_{1} \prod_{i=0, i \neq 1}^{k} y_{n-i}+\cdots+\left(a c_{k}+b\right) \prod_{i=0}^{k-1} y_{n-i}}+\cdots+\frac{c_{n-i}}{a c_{0} \prod_{i=1}^{k} y_{n-i}+a c_{1} \prod_{i=0, i \neq 1}^{k} y_{n-i}+\cdots+\left(a c_{k}+b\right) \prod_{i=0}^{k-1} y_{n-i}}
\end{gathered}
$$


and so,

$$
\begin{gathered}
y_{n+1} \leq \frac{c_{0} \prod_{i=1}^{k} y_{n-i}}{a c_{0} \prod_{i=1}^{k} y_{n-i}}+\frac{c_{1} \prod_{i=0, i \neq 1}^{k} y_{n-i}}{a c_{1} \prod_{i=0, i \neq 1}^{k} y_{n-i}}+\cdots+\frac{c_{k} \prod_{i=0}^{k-1} y_{n-i}}{\left(a c_{k}+b\right) \prod_{i=0}^{k-1} y_{n-i}} \\
=\frac{k}{a}+\frac{c_{k}}{\left(a c_{k}+b\right)}=\frac{k\left(a c_{k}+b\right)+a c_{k}}{a\left(a c_{k}+b\right)} . \\
y_{n+1}=\frac{1}{x_{n+1}} \leq \frac{k\left(a c_{k}+b\right)+a c_{k}}{a\left(a c_{k}+b\right)} .
\end{gathered}
$$

It follows that

$$
\frac{1}{x_{n+1}} \leq \frac{k\left(a c_{k}+b\right)+a c_{k}}{a\left(a c_{k}+b\right)}=E \text { for all } n \geq 1
$$

Thus we obtain

$$
x_{n}=\frac{1}{y_{n}} \geq \frac{1}{E}=m \text { for all } n \geq 1 .
$$

From (4.1) and (4.2) we see that

$$
m \leq x_{n} \leq M \quad \text { for all } n \geq 1 .
$$

Therefore every solution of Equation (1.1) is bounded.

\section{Global Stability of Equation (1.1)}

Our aim in this section we investigate the global asymptotic stability of Equation (1.1).

Theorem 5.1. If $a G=2 a c_{k}+b$, then the equilibrium point $\bar{x}$ of Equation (1.1) is global attractor.

Proof. Let $f:(0, \infty)^{k+1} \rightarrow(0, \infty)$ be a function defined by

$$
f\left(u_{0}, u_{1}, \cdots, u_{k}\right)=a+\frac{b u_{k}}{\sum_{i=0}^{k} c_{i} u_{i}},
$$

then we can see that the function $f\left(u_{0}, u_{1}, \cdots, u_{k}\right)$ is decreasing in the rest of arguments and increasing in $u_{k}$.

Suppose that $(m, M)$ is a solution of the system

$$
m=f(M, M, M, \cdots, M, m) \text { and } M=f(m, m, m, \cdots, m, M) .
$$

Then from Equation (2.1), we see that

$$
\begin{aligned}
m= & a+\frac{b m}{\sum_{i=0}^{k-1} c_{i} M+c_{k} m}, \quad M=a+\frac{b M}{\sum_{i=0}^{k-1} c_{i} m+c_{k} M}, \\
m= & a+\frac{b m}{\left(G-c_{k}\right) M+c_{k} m}, \quad M=a+\frac{b M}{\left(G-c_{k}\right) m+c_{k} M}, \\
& \left(G-c_{k}\right) M m+c_{k} m^{2}=a\left(G-c_{k}\right) M+a c_{k} m+b m, \\
& \left(G-c_{k}\right) M m+c_{k} M^{2}=a\left(G-c_{k}\right) m+a c_{k} M+b M,
\end{aligned}
$$

then

$$
c_{k}\left(m^{2}-M^{2}\right)=\left(2 a c_{k}+b-a G\right)(m-M) .
$$

Thus

$$
m=M \text {. }
$$

It follows by Theorem (1.1) that $\bar{x}$ is a global attractor of Equation (1.1) and then the proof is complete. 


\section{Numerical Examples}

For confirming the results of this section, we consider numerical examples which represent different types of solution of Equation (1.1).

Example 6.1. Consider the difference equation

$$
x_{n+1}=0.5+\frac{0.9 x_{n-1}}{0.6 x_{n}+0.5 x_{n-1}},
$$

where $k=1, a=0.5, b=0.9, \alpha=c_{0}=0.6, \beta=c_{1}=0.5$. Figure 1 shows that the equilibrium point of Equation (1.1) has locally stable, with initial data $x_{-1}=2.2, x_{0}=0.3$ (see Table 1).

Example 6.2. Consider the difference equation

$$
x_{n+1}=0.125+\frac{4 x_{n-1}}{2 x_{n}+x_{n-1}},
$$

where $k-o d d=1, a=0.125, b=4, \alpha=c_{0}=2, \beta=c_{1}=1$. Figure 2, shows that Equation (1.1) which is periodic with period two. Where the initial data satisfies condition (3.1) of Theorem (3.1) $x_{-1}=0.1, x_{0}=0.3$ (see Table 2).

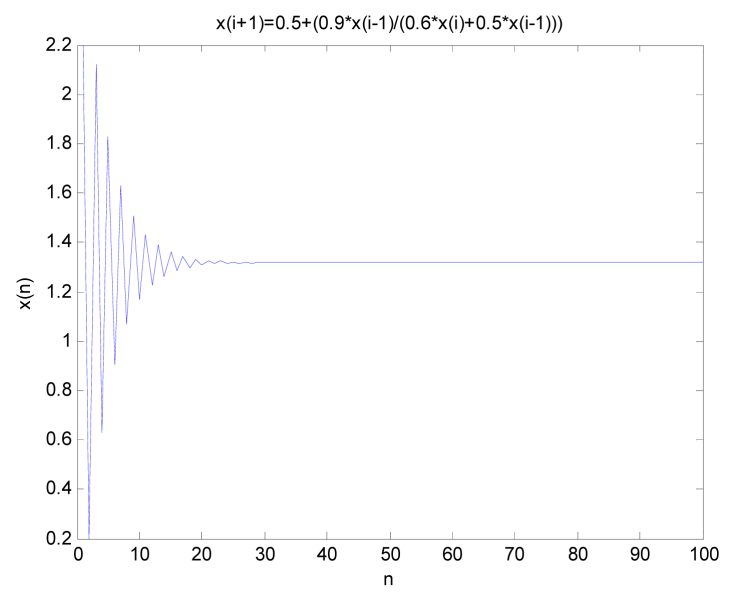

Figure 1. Ref. b1.

\begin{tabular}{|c|c|c|c|c|c|c|c|}
\hline $\mathrm{n}$ & $x(n)$ & $\mathrm{n}$ & $x(n)$ & $\mathrm{n}$ & $x(n)$ & $\mathrm{n}$ & $x(n)$ \\
\hline 1 & 2.0000 & 16 & 1.2953 & 31 & 1.3187 & 46 & 1.3182 \\
\hline 2 & 0.5000 & 17 & 1.3359 & 32 & 1.3178 & 47 & 1.3182 \\
\hline 3 & 1.8846 & 18 & 1.3044 & 33 & 1.3185 & 48 & 1.3182 \\
\hline 4 & 0.8259 & 19 & 1.3288 & 34 & 1.3179 & 49 & 1.3182 \\
\hline 5 & 1.6796 & 20 & 1.3099 & 35 & 1.3184 & 50 & 1.3182 \\
\hline 6 & 1.0232 & 21 & 1.3246 & 36 & 1.3180 & 51 & 1.3182 \\
\hline 7 & 1.5399 & 22 & 1.3132 & 37 & 1.3183 & 52 & 1.3182 \\
\hline 8 & 1.1415 & 23 & 1.3220 & 38 & 1.3181 & 53 & 1.3182 \\
\hline 9 & 1.4526 & 24 & 1.3152 & 39 & 1.3182 & 44 & 1.3182 \\
\hline 10 & 1.2123 & 25 & 1.3205 & 40 & 1.3181 & 45 & 1.3182 \\
\hline 11 & 1.3993 & 26 & 1.3164 & 41 & 1.3182 & 46 & 1.3182 \\
\hline 12 & 1.2547 & 27 & 1.3196 & 42 & 1.3182 & 57 & 1.3182 \\
\hline 13 & 1.3671 & 28 & 1.3171 & 43 & 1.3182 & 58 & 1.3182 \\
\hline 14 & 1.2801 & 29 & 1.3190 & 44 & 1.3182 & 59 & 1.3182 \\
\hline 15 & 1.3476 & 30 & 1.3175 & 45 & 1.3182 & 60 & 1.3182 \\
\hline
\end{tabular}

Table 1. The equilibrium point of Equation (1.1). 


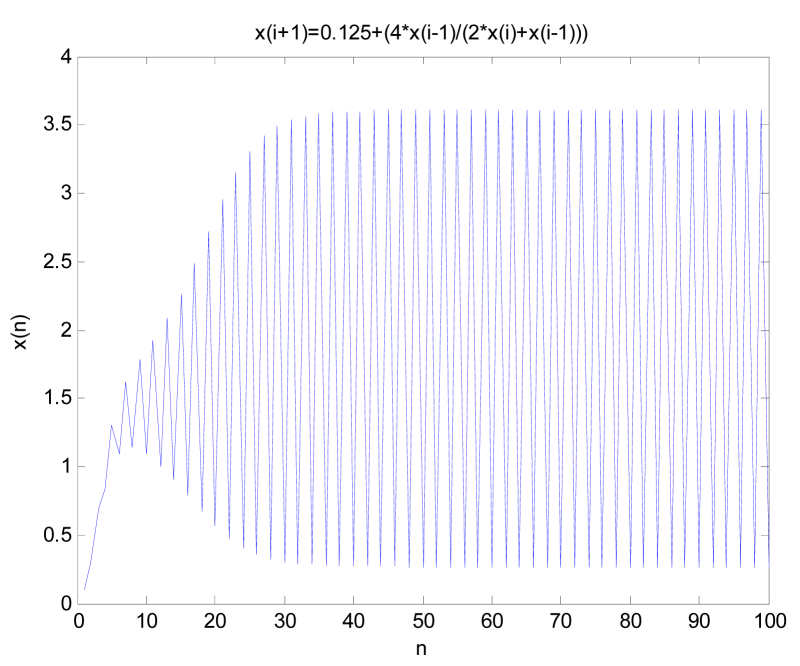

Figure 2. Ref. b4.

Table 2. The initial data satisfies condition (3.1) of Theorem (3.1).

\begin{tabular}{ccccccccccc}
\hline$n$ & $x(n)$ & $n$ & $x(n)$ & $n$ & $x(n)$ & $n$ & $x(n)$ & $n$ & $x(n)$ \\
\hline 1 & 0.1000 & 17 & 2.4834 & 33 & 3.5629 & 49 & 3.6057 & 65 & 3.6064 \\
2 & 0.3000 & 18 & 0.6734 & 34 & 0.2809 & 50 & 0.2688 & 66 & 0.2686 \\
3 & 0.6964 & 19 & 2.7184 & 35 & 3.5803 & 51 & 3.6060 & 67 & 3.6064 \\
4 & 0.8339 & 20 & 0.5658 & 36 & 0.2760 & 52 & 0.2687 & 68 & 0.2686 \\
5 & 1.3033 & 21 & 2.9493 & 37 & 3.5907 & 53 & 3.6061 & 69 & 3.6064 \\
6 & 1.0945 & 22 & 0.4751 & 38 & 0.2730 & 54 & 0.2687 & 70 & 0.2686 \\
7 & 1.6178 & 23 & 3.1503 & 39 & 3.5970 & 55 & 3.6062 & 71 & 3.6064 \\
8 & 1.1360 & 24 & 0.4055 & 40 & 0.2713 & 56 & 0.2687 & 72 & 0.2686 \\
9 & 1.7886 & 25 & 3.3061 & 41 & 3.6008 & 57 & 3.6063 & 73 & 3.6064 \\
10 & 1.0891 & 26 & 0.3561 & 42 & 0.2702 & 58 & 0.2686 & 74 & 0.2686 \\
11 & 1.9286 & 27 & 3.4160 & 43 & 3.6030 & 59 & 3.6063 & 75 & 3.6064 \\
12 & 1.0058 & 28 & 0.3232 & 44 & 0.2696 & 60 & 0.2686 & 76 & 0.2686 \\
13 & 2.0829 & 29 & 3.4886 & 45 & 3.6044 & 61 & 3.6063 & 77 & 3.6064 \\
14 & 0.9029 & 30 & 0.3021 & 46 & 0.2692 & 62 & 0.2686 & 78 & 0.2686 \\
15 & 2.2675 & 31 & 3.5345 & 47 & 3.6052 & 63 & 3.6064 & 79 & 3.6064 \\
16 & 0.7892 & 32 & 0.2889 & 48 & 0.2690 & 64 & 0.2686 & 80 & 0.2686 \\
\hline
\end{tabular}

Remark 6.1. Note that the special cases of Equation (1.1) have been studied in [9] when $k=1, b=1, c_{0}=1$, $c_{i}=0, i \geq 1$ and in [10] when $k=1, b=1, c_{0}=1, c_{i}=0, i \geq 1$ and in [11] when $b=1, c_{0}=1, c_{i}=0, i \geq 1$.

\section{References}

[1] Elabbasy, E.M., El-Metwally, H. and Elsayed, E.M. (2005) On the Periodic Nature of Some Max-Type Difference Equations. International Journal of Mathematics and Mathematical Sciences, 2005, 2227-2239. http://dx.doi.org/10.1155/IJMMS.2005.2227

[2] Elabbasy, E.M., El-Metwally, H. and Elsayed, E.M. (2006) On the Difference Equation $x_{n+1}=a x_{n}-\frac{b x_{n}}{c x_{n}-d x_{n-1}} \cdot A d-$ vances in Difference Equations, 1-10(2006), Article ID: 82579. 
[3] Elabbasy, E.M., El-Metwally, H. and Elsayed, E.M. (2007) Qualitative Behavior of Higher Order Difference Equation. Soochow Journal of Mathematics, 33, 861-873.

[4] El-Moneam, M.A. and Zayed, E. (2014) Dynamics of the Rational Difference Equation $x_{n+1}=A x_{n}+B x_{n-k}+C x_{n-l}+\frac{b x_{n} x_{n-k} x_{n-l}}{d x_{n-k}-e x_{n-l}}$. DCDIS Series A: Mathematical Analysis, 21, 317-331.

[5] Elaydi, S.N. (1996) An Introduction to Difference Equations, Undergraduate Texts in Mathematics. Springer, New York. http://dx.doi.org/10.1007/978-1-4757-9168-6

[6] Kocic, V.L. and Ladas, G. (1993) Global Behavior of Nonlinear Difference Equations of Higher Order with Applications. Kluwer Academic Publishers, Dordrecht.

[7] Stevic, S. (2005) On the Recursive Sequence $x_{n+1}=\frac{\alpha+\beta x_{n-k}}{f\left(x_{n}, \cdots, x_{n-k+1}\right)}$. Taiwanese Journal of Mathematics, 9, 583-593.

[8] Zayed, E. and EI-Moneam, M.A. (2010) On the Rational Recursive Sequence $x_{n+1}=\frac{\alpha_{0} x_{n}+\alpha_{1} x_{n-l}+\alpha_{2} x_{n-k}}{\beta_{0} x_{n}+B_{1} x_{n-l}+\beta_{2} x_{n-k}}$.Mathematica Bohemica, 135, 319-363.

[9] Amleh, A.M., Grove, E.A., Georgiou, D.A. and Ladas, G. (1999) On the Recursive Sequence $x_{n+1}=\alpha+\frac{x_{n-1}}{x_{n}}$. Journal of Mathematical Analysis and Applications, 233, 790-798. http://dx.doi.org/10.1006/jmaa.1999.6346

[10] Hamza, A.E. (2006) On the Recursive Sequence $x_{n+1}=\alpha+\frac{x_{n+1}}{x_{n}}$. Journal of Mathematical Analysis and Applications, 322, 668-674. http://dx.doi.org/10.1016/j.jmaa.2005.09.029

[11] Saleh, M. and Aloqeili, M. (2005) On the Rational Difference Equation $x_{n+1}=A+\frac{x_{n-k}}{x_{n}}$. Applied Mathematics and Computation, 171, 862-869. http://dx.doi.org/10.1016/j.amc.2005.01.094

[12] Grove, E.A. and Ladas, G. (2005) Periodicities in Nonlinear Difference Equations. Vol. 4, Chapman and Hall/CRC, Boca Raton.

[13] Elabbasy, E.M., El-Metwally, H. and Elsayed, E.M. (2007) On the Difference Equations $x_{n+1}=\frac{\alpha x_{n-k}}{\beta+\gamma \prod_{i=0}^{k} x_{n-i}}$. Journal of Concrete and Applicable Mathematics, 5, 101-113.

[14] Kulenovic, M.R.S. and Ladas, G. (2001) Dynamics of Second Order Rational Difference Equations with Open Problems and Conjectures. Chapman \& Hall/CRC, Florida. http://dx.doi.org/10.1201/9781420035384 